Research Article

\title{
Acceptability of COVID-19 Vaccination among Health Care Workers in Ghana
}

\author{
Martin Wiredu Agyekum (D, ${ }^{1}$ Grace Frempong Afrifa-Anane ${ }^{(D)}{ }^{2}$ \\ Frank Kyei-Arthur $\mathbb{D}^{2}{ }^{2}$ and Bright Addo $\mathbb{D}^{3}$ \\ ${ }^{1}$ Regional Institute for Population Studies, University of Ghana, Legon, Accra, Ghana \\ ${ }^{2}$ Department of Environment and Public Health, University of Environment and Sustainable Development, Somanya, \\ Eastern Region, Ghana \\ ${ }^{3}$ Department of Sociology and Social Work, Kwame Nkrumah University of Science and Technology, Kumasi, Ghana
}

Correspondence should be addressed to Bright Addo; addobright1@gmail.com

Received 7 March 2021; Revised 31 March 2021; Accepted 9 April 2021; Published 20 April 2021

Academic Editor: Hamidreza Karimi-Sari

Copyright (C) 2021 Martin Wiredu Agyekum et al. This is an open access article distributed under the Creative Commons Attribution License, which permits unrestricted use, distribution, and reproduction in any medium, provided the original work is properly cited.

\begin{abstract}
Because health care workers are a reliable source of health information, their acceptance or rejection of COVID-19 vaccines can influence the general population's uptake of COVID-19 vaccines. In this study, we sought to determine the acceptability of COVID-19 vaccines among health care workers in Ghana. Using a cross-sectional design, we collected data from 234 health care workers through a self-administered online survey from 16 January to 15 February 2021. Descriptive, bivariate, and multivariate analyses were performed using STATA version 15. The findings revealed that $39.3 \%(n=92)$ of health care workers intended to receive the COVID-19 vaccines. Factors such as sex $(\mathrm{AOR}=0.451$; CI 95\% 0.240-0.845; $p=0.013)$, category of health care workers $(\mathrm{AOR}=2.851 ; 95 \mathrm{CI} \%: 1.097-7.405 ; p=0.031)$, relative being diagnosed with COVID-19 (AOR=0.369; CI 95\% $0.145-0.941 ; p=0.037)$, and trust in the accuracy of the measures taken by the government in the fight against COVID-19 $(\mathrm{AOR}=2.768$; CI 95\%: $1.365-5.616 ; p=0.005)$ proved to be significant predictors of the acceptability of the COVID-19 vaccine. Concerns about the safety of vaccines $(n=93,65.5 \%)$ and the adverse side effects of the vaccines $(n=23,14.8 \%)$ were identified as the main reasons why health care workers would decline uptake of COVID-19 vaccines in Ghana. The self-reported low intention of health care workers in Ghana to accept COVID-19 vaccines necessitates an urgent call from the Government of Ghana and other stakeholders to address health care workers' concerns about the safety and adverse side effects of COVID-19 vaccines, as this would increase vaccine uptake. Interventions must also take into consideration sex and the category of health care workers to achieve the desired results.
\end{abstract}

\section{Introduction}

In March 2020, the novel coronavirus disease (COVID-19) was declared a global pandemic after its emergence in Wuhan, China, in November 2019. Globally, as of 28 March 2021, there were 126,359,540 confirmed cases of COVID-19 and $2,769,473$ deaths [1]. In Ghana, the total number of confirmed cases as of 24 March was 90,287 with 740 deaths [2]. Ghanaian health care workers have not been spared the brunt of the pandemic. Since the emergence of COVID-19 in Ghana on 12 March 2020, approximately 1,629 nurses and midwives have been infected with the virus and 4 have died as of 14 February 2021 [3]. Also, more than 450 doctors and dentists have been infected with COVID-19 and 7 have died [4].

Governments globally have imposed several measures and protocols to help halt the spread of COVID-19 such as travel bans, wearing of nose masks, lockdowns, social distance, and frequent washing of hands with soap and water, among others. To further halt the spread and deaths associated with COVID-19, researchers and pharmaceutical companies are collaborating to develop safe and effective vaccines for mass vaccination. Vaccination is an effective way of combating 
infectious conditions [5]. As of 26 March 2021, 83 vaccines were in the clinical development stage while 184 were at the pre-clinical development stage [6]. Globally, several vaccines have been deemed safe and effective for human use, including Pfizer, Oxford/AstraZeneca, Moderna, Janssen, Sputnik V, Sinovac, and Sinopharm [7-10].

Due to the inadequate supply of COVID-19 vaccines globally, governments have prioritised high-risk groups to receive the initial supply of vaccines. These high-risk groups include health care workers, older persons especially those with chronic co-morbid conditions, and those in essential services [11]. Health care workers are at a high risk of contracting the COVID-19 disease due to their direct or indirect contact with bodily secretions, COVID-19 patients/ clients, visitors, and other health care workers who may have been exposed to COVID-19 [12, 13].

The availability of COVID-19 vaccines may not translate into its uptake. Although governments will provide the vaccines, their uptake is voluntary [7]. Indeed, several studies have demonstrated that not all health care workers are ready to accept COVID-19 vaccines when made available in their country [14-16]. For example, a study conducted in the Democratic Republic of Congo found that approximately $28 \%$ of health care workers were willing to receive the COVID-19 vaccine if available [17]. Reasons for hesitancy to accept COVID-19 vaccines have been identified to include concerns over vaccine safety and side effects and speed of vaccine development/approval [18-20].

The Government of Ghana (GoG) has publicly articulated its decision to initiate the procurement of the Oxford/AstraZeneca and Sputnik V COVID-19 vaccines for use in the country. Health care workers have been identified as the first recipients of the vaccine. Since the announcement was made for the purchase of COVID-19 vaccines in Ghana, there has been a mixed response as to whether or not to accept or refuse vaccination by several people in the country, including health care workers. Limited studies have been conducted in Ghana to determine the extent to which health care workers will accept vaccination.

Health care workers are a reliable source of information on vaccination to patients $[14,21]$; therefore their acceptance or otherwise of COVID-19 vaccines may influence the uptake of COVID-19 among the general population. People commonly rely on health care workers information and actions to guide their decision. For instance, studies have shown that health care workers counselling on exclusive breastfeeding and complementary feeding influence the practice of exclusive breastfeeding among mothers $[22,23]$. Against this backdrop, this study sought to assess and identify the determinants of COVID-19 acceptability among health care workers in Ghana. Examining the acceptance of COVID-19 vaccination among health workers would help researchers and policymakers to design appropriate interventions to reduce vaccine hesitancy among health workers and the general population.

\section{Methods}

2.1. Study Setting. Ghana is a lower-middle-income country and it has an estimated 30.9 million population. It is bounded by Burkina Faso on the North, the Gulf of Guinea on the South, Togo on the East, and Cote d'Ivoire on the West. Ghana has 16 administrative regions. As of 2017, Ghana had 5,421 Community-based Health Planning and Services (CHPS), 998 clinics, 140 district hospitals, 1004 health centres, and 357 hospitals. Health facilities and health workers in Ghana are disproportionately found in the Ashanti and Greater Accra regions, especially in urban areas. For instance, out of the 357 hospitals in Ghana, 128 (35.9\%) are found in the Ashanti region and 99 (27.7\%) in the Greater Accra region [24]. Also, Ghana has 4,016 doctors as of 2017 and almost two-fifths (39.4\%) are found in the Greater Accra region while one-fifth (20.5\%) are found in the Ashanti region. In terms of critical health staff (such as community health nurses, enrolled nurses, medical officers, and pharmacist, among others), Ghana has 90,703 personnel, out of which 13,438 (14.8\%) and 13,120 (14.5\%), respectively, are found in Ashanti and Greater Accra regions [24].

2.2. Study Design. This survey is a cross-sectional study conducted among health care workers in Ghana using both convenient and snowballing sampling techniques. Google Form was used to design an online self-administered questionnaire and it was disseminated through WhatsApp to health care workers' platforms. In addition, a snowball sampling technique was used to reach out to health workers by encouraging respondents to forward or share the online survey link to other health workers. This approach was adopted because of the existing nature of the pandemic, as it offers appropriate social distancing and excludes movements of researchers or participants [25]. To be included in the study, the individual must be a health professional working in a registered health care setting and must consent and be willing to participate in the survey. Participation in the study was voluntary and anonymity was ensured. The data were collected from 16 January 2021 to 15 February 2021. The study observed all ethical guidelines in relation to the collection of data from human subjects. Ethical approval was sought and received from the University of Environment and Sustainable Development Ethics Committee. All participants gave written consent.

2.3. Measurement and Variables. Acceptance in this study was defined as the intention to accept to take the COVID-19 vaccines. This was the dependent variable and it was assessed using the question: "if an approved COVID-19 vaccine becomes available, would you take it yourself?" and the responses are "Yes" and "No."

The questionnaire developed covered four sections: (i) sociodemographic characteristics, (ii) COVID-19 experience, (iii) trust in the fight against COVID-19 pandemic, and (iv) intention to vaccinate. The sociodemographic characteristics included measures such as age, sex, category of health care workers and chronic illness status, among others. In addition, respondents were asked questions on their COVID-19 experience, including previous contact with a COVID-19 patient, whether a member of their household, relatives, friends, or neighbours has been diagnosed with 
COVID-19. To measure social trust in the fight against COVID-19 pandemic, nine questions were used and assessed using a 5-point Likert scale (strongly disagree, disagree, neutral, agree, and strongly agree) (Supplementary file).

These nine questions have been validated for the measure in previous studies [26]. However, some of the questions were modified to reflect our local context. Confirmatory factor analysis was performed to evaluate the reliability validity of the Scale. A Cronbach's alpha coefficient was used to check for the reliability of the social trust scale. The Cronbach alpha coefficient of the scale was 0.8675 . A Cronbach alpha coefficient higher than 0.60 indicates high reliability [26].

2.4. Statistical Analysis. The data were analysed using STATA version 15. Descriptive statistics were used to describe the sociodemographic characteristics of health workers. Also, Pearson's chi-square was used to determine the association between intention to vaccinate and sociodemographic characteristics, COVID-19 experience, and social trust in the fight against the COVID-19 pandemic. The dependent variable was dichotomous so multivariate binary logistic regression analysis was used to identify the determinants of intention to vaccinate. All variables were considered statistically significant at $95 \%$ confidence interval $(p<0.05)$.

\section{Results}

3.1. Sample Characteristics. There were a total of 234 health care workers who completed the online survey. Of the 234 health care workers, more than half $(n=131,56 \%)$ were aged 30-39 years. The majority of the health care workers were females $(n=148,63.2 \%)$, Christians $(n=216,92.3 \%)$, and living in an urban area $(n=179,76.5 \%)$. A higher proportion of females $(n=99,66.9 \%)$ significantly indicated non-acceptance of COVID-19 vaccine compared to males $(n=43$, $50 \% ; p<0.05)$.

Regarding educational attainment, the highest proportion $(n=86,36.8 \%)$ of health care workers had a bachelor's degree. Also, the majority $(n=151,64.5 \%)$ of health care workers were nurses and midwives. In terms of marital status, $43.2 \%$ of the health care workers were never married and a higher proportion of those who were never married $(n=70,69.3 \%)$ indicated non-acceptance of the COVID-19 vaccine compared to those who were married $(n=72,54.1 \%$; $p<0.05)$. About four-fifths $(79.1 \%)$ of the health care workers had received vaccination in their lifetime and $90.6 \%$ of the health care workers reported no chronic disease (Table 1).

3.2. Acceptance ofCOVID-19 Vaccines. Out of the 234 health care workers who participated in the study, about two-fifths $(n=92,39.3 \%)$ of them indicated acceptance of COVID-19 vaccines if available while more than half $(n=142,60.7 \%)$ indicated non-acceptance of COVID-19 vaccines if available.
3.3. Health Care Workers Experience with COVID-19. About $60.7 \%$ of the respondents indicated that they had not been in contact with any COVID-19 patient, and a higher proportion of those who had not been in contact with a COVID-19 patient $(n=94,66.2 \%)$ indicated no acceptance of the COVID-19 vaccines compared to those who had contacted a patient $(n=48,52.2 \% ; p<0.05)$. Also, eight out of ten $(83.8 \%)$ respondents indicated that no member in their households had been diagnosed with COVID-19 and a higher proportion of those whose household member(s) had been diagnosed with COVID-19 $(n=22,57.9 \%)$ indicated acceptance of the COVID-19 vaccines $(p<0.05)$. In addition, $68.8 \%$ of the respondents had none of their relatives been diagnosed with COVID-19, and the highest proportion of health care workers $(n=104,44.4 \%)$ indicated that their friends had been diagnosed with COVID-19. Approximately $48 \%$ of the respondents had been tested for COVID-19. Also, $47.9 \%$ of the respondents indicated a great chance of getting COVID-19 and the majority of the study respondents $(n=213,91 \%)$ had heard of the COVID-19 vaccines (Table 2).

3.4. Social Trust and Acceptance of COVID-19 Vaccines. The results of the study showed that about $42.3 \%$ of health care workers trusted the authorities in the fight against COVID-19, and $58.1 \%$ had trust in the information provided by the media in the fight against COVID-19. Also, about half ( $n=114,48.7 \%)$ of the health care workers had trust in the health care system and hospitals in the fight against COVID19 , and $45.7 \%$ had trust in the correct implementation of the measures to combat COVID-19. In addition, about $39.3 \%$ of health care workers trusted the appropriateness of the economic measures taken with COVID-19 while a higher proportion ( $n=93,39.8 \%)$ disagreed with the statement that Ghana is more successful than the western countries in the fight against COVID-19. Furthermore, more than half $(n=132,56.4 \%)$ of health care workers disagreed that the COVID-19 pandemic will lose its effect with sunshine (Table 3).

3.5. Factors Associated with COVID-19 Vaccines Acceptance. Factors associated with acceptance of COVID-19 vaccines among the health care workers are presented in Table 4. Only variables that were significant at the bivariate level were included in the model. The results showed that sex, category of health care workers, relatives been diagnosed with COVID-19, and trust in the accuracy of measures taken by the government in the fight against COVID-19 were significantly associated with acceptance of the COVID-19 vaccine. Female health care workers were less likely to accept COVID-19 vaccine than males $(\mathrm{AOR}=0.451$; CI $95 \%$ $0.240-0.845 ; p=0.013)$. Also, medical doctors were more likely to accept the COVID-19 vaccines than nurses and midwife $(\mathrm{AOR}=2.851 ; 95 \mathrm{CI} \%$ : 1.097-7.405; $p=0.031)$. Health care workers who indicated that they do not know if their relatives had been diagnosed with COVID-19 were less likely to accept the COVID-19 vaccines compared with those whose relatives had been diagnosed with COVID-19 
TABLE 1: Background characteristics of health care workers.

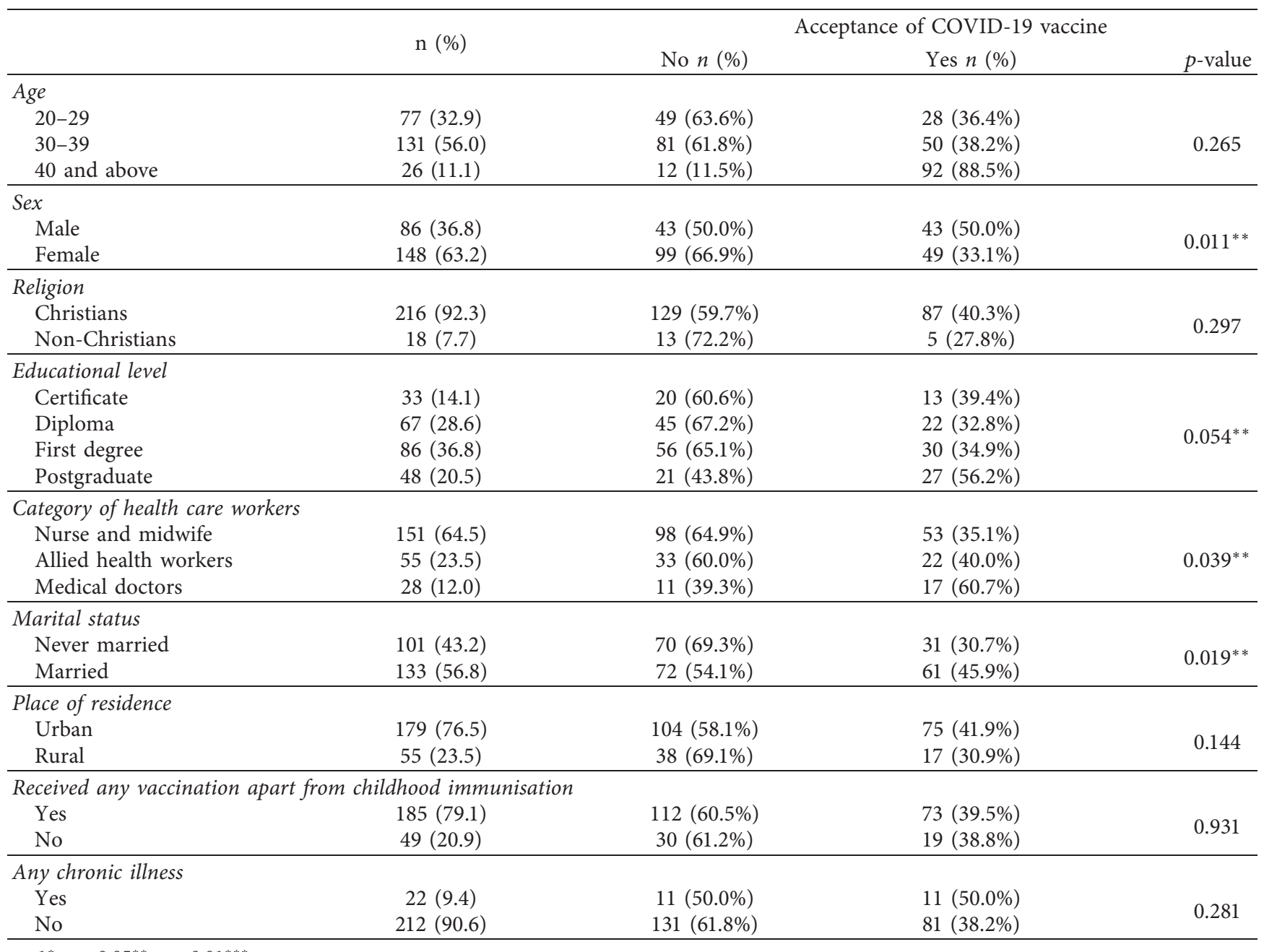

$p<1^{*}, p<0.05^{* *}, p<0.01^{* * *}$.

$(\mathrm{AOR}=0.369 ; \mathrm{CI} 95 \% 0.145-0.941 ; p=0.037)$. Health care workers who agreed to the accuracy of measures taken by the government to fight COVID-19 were more likely to accept the COVID-19 vaccines than those who had no trust in the accuracy of the measures taken by the government in the fight against COVID-19 (AOR = 2.768; CI 95\%: 1.365-5.616; $p=0.005)$.

3.6. Reasons for Non-Acceptance of COVID-19 Vaccines. The majority $(64.5 \%)$ of health care workers were unwilling to accept the COVID-19 vaccines due to concern about the safety of the vaccines. Also, about $16 \%$ and $5 \%$ of them were unwilling to accept the COVID-19 vaccines due to concern after adverse effects of the vaccine, and acquiring COVID-19 through the vaccination, respectively (Table 5).

\section{Discussion}

This study examined the acceptability of COVID-19 vaccines among health care workers in Ghana. The findings showed that approximately $39 \%$ of health care workers in Ghana intended to receive the COVID-19 vaccines if available. The acceptability of the COVID-19 vaccines in this study is comparable to that of Wang et al. [20] who found that about $40 \%$ of nurses in Hong Kong had the intention to accepting the COVID-19 vaccine if available. Similarly, in the multicountry study of Verger et al. [16], which also assessed health care workers' attitudes towards COVID-19 vaccination in France, Belgium, and Canada, it was found that approximately $40 \%$ of health care workers in Belgium (Wallonia and Brussels) were willing to vaccinate themselves if COVID-19 vaccines were available.

The acceptability of the COVID-19 vaccines in this study is higher compared to other countries in the sub-Saharan Africa region. For example, the Nzaji et al. [17] study in the Democratic Republic of Congo found that approximately $28 \%$ of health care workers were willing to receive the COVID-19 vaccines if available. The authors [17] explained that the low willingness to receive COVID-19 vaccines can be attributed to misinformation on the quality of vaccines and spread of COVID-19 especially on social media. Misinformation and conspiracy theories have been identified as a barrier to the fight against COVID-19 [27]. Conversely, the acceptability of the COVID-19 vaccines among health care workers in this study was lower than that reported in Saudi 
TABLE 2: COVID-19 experience of health care workers.

\begin{tabular}{|c|c|c|c|c|}
\hline & \multirow{2}{*}{ n (\%) } & \multicolumn{3}{|c|}{ Acceptance of COVID-19 vaccine } \\
\hline & & No $n(\%)$ & Yes $n(\%)$ & $p$-value \\
\hline \multicolumn{5}{|l|}{ Contact with COVID-19 patient } \\
\hline Yes & $92(39.3)$ & $48(52.2 \%)$ & $44(47.8 \%)$ & \multirow{2}{*}{$0.032 * *$} \\
\hline No & $142(60.7)$ & $94(66.2 \%)$ & $48(33.8 \%)$ & \\
\hline \multicolumn{5}{|c|}{ Member of household diagnosed with COVID-19 } \\
\hline Yes & $38(16.2)$ & $16(42.1 \%)$ & $22(57.9 \%)$ & \multirow{2}{*}{$0.010 * *$} \\
\hline No & $196(83.8)$ & $126(64.3 \%)$ & $70(35.7 \%)$ & \\
\hline \multicolumn{5}{|l|}{ Relatives have been diagnosed with COVID-19 } \\
\hline Yes & $45(19.2)$ & $18(40.0 \%)$ & $27(60.0 \%)$ & \multirow{3}{*}{$0.006 * *$} \\
\hline No & $161(68.8)$ & $107(66.5 \%)$ & $54(33.5 \%)$ & \\
\hline Do not know & $28(12.0)$ & $17(60.7 \%)$ & $11(39.3 \%)$ & \\
\hline \multicolumn{5}{|l|}{ Friends have been diagnosed with COVID-19 } \\
\hline Yes & $104(44.4)$ & $55(52.9 \%)$ & $49(47.1 \%)$ & \multirow{3}{*}{$0.078 *$} \\
\hline No & $95(40.6)$ & $65(68.4 \%)$ & $30(31.6 \%)$ & \\
\hline Do not know & $35(15.0)$ & $22(62.9 \%)$ & $13(37.1 \%)$ & \\
\hline \multicolumn{5}{|l|}{ Have tested for COVID-19 } \\
\hline Yes & $113(48.3)$ & $69(61.1 \%)$ & $44(38.9 \%)$ & \multirow{2}{*}{0.909} \\
\hline No & $121(51.7)$ & $73(60.3 \%)$ & $48(39.7 \%)$ & \\
\hline \multicolumn{5}{|l|}{ Results of COVID test } \\
\hline Positive & $22(19.5)$ & $12(54.5 \%)$ & $10(45.5 \%)$ & \multirow{3}{*}{0.781} \\
\hline Negative & $80(70.8)$ & $50(62.5 \%)$ & $30(37.5 \%)$ & \\
\hline Do not know/did not receive the result & $11(9.7)$ & $7(63.6 \%)$ & $4(36.4 \%)$ & \\
\hline \multicolumn{5}{|l|}{ Chance of getting COVID-19 } \\
\hline Small & $37(15.8)$ & $26(70.3 \%)$ & $11(29.7 \%)$ & \multirow{4}{*}{0.348} \\
\hline Moderate & $70(29.9)$ & $39(55.7 \%)$ & $31(44.3 \%)$ & \\
\hline Great & $112(47.9)$ & $66(58.9 \%)$ & $46(41.1 \%)$ & \\
\hline No risk at all & $15(6.4)$ & $11(73.3 \%)$ & $4(26.7 \%)$ & \\
\hline \multicolumn{5}{|l|}{ Heard about the COVID-19 vaccine } \\
\hline Yes & $213(91.0)$ & $130(61.0 \%)$ & $83(39.0 \%)$ & \multirow[b]{2}{*}{0.728} \\
\hline No & $21(9.0)$ & $12(57.1 \%)$ & $9(42.9 \%)$ & \\
\hline
\end{tabular}

Arabia [28], China [29, 30], France [31], and the United States $[14,19,32]$. For instance, Shaw et al. [14] study in the United States found that about $58 \%$ of health care workers had the intention to receive COVID-19 vaccines if available. The high acceptability of COVID-19 vaccines in these countries can be attributed to high publicity on potential COVID-19 vaccines in these countries. For instance, Barry et al. [28] acknowledged that their study in Saudi Arabia was conducted during the period of high publicity on potential COVID-19 vaccines, which could have influenced the attitudes and perceptions of health care workers. Also, Gagneux-Brunon et al.'s [31] study in France was conducted during the first pandemic wave. The finding of low acceptance of the COVID-19 vaccines in the present study is worrying considering the associated consequences. Research has shown that health care workers intending to be vaccinated plan to recommend the vaccines to family and friends [18]. This suggests that low acceptance of the COVID-19 vaccine among health care workers may have a negative impact on the general population.

We also found that sex, category of health care workers, relative having been diagnosed of COVID-19, and trust in the accuracy of the measures taken by the government in the fight against COVID-19 predicted acceptability of COVID-
19 vaccination among health care workers in Ghana. The findings also revealed that female health care workers were less likely to accept the COVID-19 vaccines if available compared to males. This finding corroborates other empirical studies that indicate that male health care workers are more likely to accept COVID-19 vaccines compared to female health care workers $[14,17,18,31]$. The higher likelihood for male health care workers to accept COVID-19 vaccination has been attributed to increased risk perception of the disease in men compared to women [17].

Medical doctors were more likely to accept the COVID19 vaccines if available compared to nurses and midwives. This finding is consistent with other studies that found that nurses are less likely to accept COVID-19 vaccines compared to doctors $[14,15,17,31]$. According to GagneuxBrunon et al. [31], the majority of nurses and midwives being females could explain why nurses and midwives are less likely to accept COVID-19 vaccines if available compared to medical doctors.

The findings also showed that health care workers whose relatives have not been diagnosed with COVID-19 were less likely to accept the COVID-19 vaccines if available compared to health care workers whose relatives have been diagnosed with COVID-19. A probable explanation is that 
TABLE 3: Social trust and acceptance of COVID-19 vaccine.

\begin{tabular}{ccccc}
\hline \multirow{2}{*}{$\mathrm{n}(\%)$} & \multicolumn{3}{c}{ Acceptance of COVID-19 vaccine } \\
& No $n(\%)$ & Yes $n(\%)$ & $p$-value \\
\hline I trust in the authorities in the fight against COVID-19 \\
Disagree & $64(27.4)$ & $40(62.5 \%)$ & $24(37.5 \%)$ \\
Neutral & $71(30.3)$ & $45(63.4 \%)$ & $26(36.6 \%)$ & 0.703 \\
Agree & $99(42.3)$ & $57(57.6 \%)$ & $42(42.4 \%)$ &
\end{tabular}

I trust in the information provided by the media in the fight against COVID-19

$\begin{array}{lcccc}\text { Disagree } & 44(18.8) & 28(63.6 \%) & 16(36.4 \%) & \\ \text { Neutral } & 54(23.1) & 36(66.7 \%) & 18(33.3 \%) & 0.449 \\ \text { Agree } & 136(58.1) & 78(57.4 \%) & 58(42.6 \%) & \end{array}$

I trust in our health system and hospitals in the fight against COVID-19

\begin{tabular}{lcccc} 
Disagree & $65(27.8)$ & $45(69.2 \%)$ & $20(30.8 \%)$ & \\
Neutral & $55(23.5)$ & $36(65.4 \%)$ & $19(34.6 \%)$ & $0.083 *$ \\
Agree & $114(48.7)$ & $61(53.5 \%)$ & $53(46.5 \%)$ & \\
\hline
\end{tabular}

I trust in the accuracy of the measures taken by the government in the fight against COVID-19

$\begin{array}{lcccc}\text { Disagree } & 76(32.6) & 51(67.1 \%) & 25(32.9 \%) & \\ \text { Neutral } & 47(20.0) & 33(70.2 \%) & 14(29.8 \%) & 0.041 * * \\ \text { Agree } & 111(47.4) & 58(52.2 \%) & 53(47.8 \%) & \end{array}$

I trust in the correct implementation of the measures in the fight against COVID-19

$\begin{array}{lcccc}\text { Disagree } & 72(30.8) & 38(52.8 \%) & 34(47.2 \%) & \\ \text { Neutral } & 55(23.5) & 37(67.3 \%) & 18(32.7 \%) & 0.217 \\ \text { Agree } & 107(45.7) & 67(62.6 \%) & 40(37.4 \%) & \end{array}$

I trust in the appropriateness of the economic measures taken with respect to COVID-19

Disagree $71(30.3) \quad 39(54.9 \%) \quad 32(45.1 \%)$

Neutral $\quad 71(30.3) \quad 50(70.4 \%) \quad 21(29.6 \%)$

Agree $\quad 92(39.4) \quad 53(57.6 \%) \quad 39(42.4 \%)$

I think that we are more successful than the western countries in the fight against COVID-19

Disagree $93(39.8) \quad 54(58.1 \%) \quad 39(41.9 \%)$

Neutral $\quad 63(26.9) \quad 42(66.7 \%) \quad 21(33.3 \%)$

Agree $\quad 78(33.3) \quad 46(59.0 \%) \quad 32(41.0 \%)$

I think that sunshine will make COVID-19 lose its effect

Disagree $132(56.4) \quad 75(56.8 \%) \quad 57(43.2 \%)$

Neutral $\quad 62(26.5) \quad 42(67.7 \%) \quad 20(32.3 \%)$

Agree $\quad 40(17.1) \quad 25(62.5 \%) \quad 15(37.5 \%)$

$p<1^{*}, p<0.05^{* *}$, and $p<0.01^{* * *}$.

health care workers whose relatives have been diagnosed with COVID-19 may have gained more knowledge about COVID-19 and its effects on human health and consequently, they may want to protect themselves if the COVID19 vaccines become available.

Furthermore, the study found that health care workers who had trust in the accuracy of the measures taken by the government in the fight against COVID-19 were more likely to accept COVID-19 vaccines if available. During mass vaccination programmes, trust in authorities is key. The Strategic Advisory Group of Experts of the World Health Organization have identified trust in governments by citizens as essential for vaccine confidence as this helps reduce vaccine hesitancy [33]. The trust in the accuracy of other measures taken by the government of Ghana so far in the fight against COVID-19 disease may have influenced some
TABLE 4: Logistic regression model for intention to accept COVID19 vaccine.

\begin{tabular}{|c|c|c|}
\hline & \multicolumn{2}{|c|}{$R^{2}=0.1175$} \\
\hline & AOR (95\% C.I) & p-value \\
\hline \multicolumn{3}{|l|}{ Sex } \\
\hline $\begin{array}{l}\text { Male } \\
\text { Female }\end{array}$ & $\begin{array}{c}\text { Ref } \\
0.45(0.240-0.845)\end{array}$ & $0.013 * *$ \\
\hline \multicolumn{3}{|c|}{ Category of health care workers } \\
\hline Nurses and midwife & Ref & \\
\hline Allied health workers & $0.93(0.458-1.895)$ & 0.845 \\
\hline Medical doctors & $2.85(1.097-7.405)$ & $0.031 * *$ \\
\hline \multicolumn{3}{|l|}{ Marital status } \\
\hline Never married & Ref & \\
\hline Married & $1.49(0.824-2.683)$ & 0.188 \\
\hline \multicolumn{3}{|c|}{ Contact with COVID-19 patient } \\
\hline Yes & Ref & \\
\hline No & $0.89(0.462-1.728)$ & 0.738 \\
\hline \multicolumn{3}{|c|}{ Member of household diagnosed with COVID-19 } \\
\hline Yes & Ref & \\
\hline No & $0.68(0.261-1.777)$ & 0.432 \\
\hline \multicolumn{3}{|c|}{ Relatives have been diagnosed with COVID-19 } \\
\hline Yes & Ref & \\
\hline No & $0.37(0.145-0.941)$ & $0.037 * *$ \\
\hline Do not know & $0.56(0.172-1.839)$ & 0.341 \\
\hline
\end{tabular}

I trust in the accuracy of the measures taken by the government in the fight against COVID-19

Disagree

Neutral

Ref

Agree

$0.83(0.344-2.020)$

$2.77(1.364-5.616)$

0.686

Constant

$1.45(0.494-4.252)$

$0.005 * *$

0.499

Only variables with a $p<0.05$ in univariate analysis were integrated into the model. Ref, reference. $p<1^{*}, p<0.05^{* *}$, and $p<0.01^{* * *}$.

health care workers to be willing to accept COVID-19 vaccines if they become available. Therefore, it is important for the government and health authorities to remain transparent to health care workers regarding measures in the fight against COVID-19 disease to strengthen trust between them and health care workers.

The study also revealed that concerns about the safety of vaccines and adverse side effects of the vaccine were the main reasons why health care workers were unwilling to accept the COVID-19 vaccines. These findings are consistent with other studies $[14,15,18,19]$. For instance, a study in the Kingdom of Saud Arabia found concerns about the safety of vaccines and concern about side effects as the main reasons for unwillingness to accept COVID-19 vaccines [28]. The attitude and uptake of vaccination by health professionals is associated with the acceptance of vaccination by patients and compliance with vaccination schedules and thus reduces aversion or hesitation towards vaccination [17]. To enhance the acceptability of COVID-19 vaccination among health care workers and the general population in Ghana, policymakers in the health sector should address the concerns of health care workers about the safety and side effects of the COVID-19 vaccines as early as possible. Also, policymakers in the health sector should use both traditional and social media to strengthen public education on COVID-19 and the 
TABLE 5: Reasons for non-acceptance of COVID-19 vaccines $(N=142)$.

\begin{tabular}{lr}
\hline Reasons for not taking COVID-19 vaccine & $n(\%)$ \\
\hline Inadequate data about the safety of the vaccine & $93(65.5)$ \\
A concern on the adverse effects of the vaccine & $23(14.8)$ \\
A concern of acquiring COVID-19 infection & $7(4.9)$ \\
A concern on the vaccine being ineffective & $4(2.8)$ \\
I am against vaccine in general & $4(2.8)$ \\
I perceive myself not to be at considerable risk of developing complications if I am infected with COVID-19 & $4(2.8)$ \\
I already had the COVID-19 disease & $3(2.1)$ \\
Prior adverse reaction to any vaccine & $2(1.4)$ \\
Other & $4(2.8)$ \\
\hline
\end{tabular}

benefits of injecting the COVID-19 vaccines since they are the mediums through which misinformation on COVID-19 is spread especially social media.

Furthermore, interventions designed to address these concerns should be tailor-made taking into consideration the sex and category of health care workers.

\section{Limitations}

This study has some limitations. First, the study was crosssectional and therefore causality cannot be established. Second, this study used convenient and snowball sampling techniques, which are non-probability sampling techniques, thus limiting the extent to which the results can be generalized to the population of health care workers in Ghana, although the online survey link was shared on health care workers' WhatsApp platforms with members across the country. Third, health care workers could have made multiple submissions since no identification verification tool was used. Fourth, health care workers who had no access to the Internet could not participate in this study. Fifth, an online survey can lead to a low response rate and this was evident in the present study. Despite these limitations, this study highlights the determinants of COVID-19 vaccine acceptability and concerns about COVID-19 vaccines, which will help to reduce vaccine hesitancy among Ghanaian health care workers.

\section{Conclusion}

This study demonstrates that interventions to promote vaccination of COVID-19 among health care workers in Ghana should take into account their sociodemographic characteristics (such as sex and category of health care workers), COVID-19 experience, and trust in the measures taken by the government in the fight against COVID-19 to achieve the desired results.

\section{Data Availability}

The data used in this study can be obtained on written request to the corresponding author.

\section{Conflicts of Interest}

The authors declare that they have no known competing interests.

\section{Authors' Contributions}

This study was conceived and designed by FK-A, MWA, and GFA-A. FK-A, MWA, GFA-A, and BA led the data collection. The data processing and analysis were conducted by MWA. The manuscript was prepared by FK-A, MWA, GFAA, and BA. All authors contributed to the editing and final approval of the manuscript for submission.

\section{Acknowledgments}

The authors would like to thank all the health care workers who took time off their busy schedules to participate in the survey exercise. No funding was received from a funding body for this study.

\section{Supplementary Materials}

Supplementary File: questionnaire for online survey administered to health care workers. (Supplementary Materials)

\section{References}

[1] WHO, "WHO coronavirus disease (COVID-19) Dashboard," 2021, Available from: https://covid19.who.int/? gclid=CjwKCAiA65iBBhB-EiwAW253W7s4F5NSHcFwrB231 LaeQI6tq-dDxYgpVLNdfpw16xGdP3ZQw4YbtBoCoHIQAv D_BwE.

[2] Ghana Health Service, "Ghana Health Service COVID-19 Dashboard,” 2021, Available from: https://ghanahealthservice. org/covid19/.

[3] MyJoyOnline, 789 Nurses and Midwives Test Positive for Covid-19 in 2021 - General Secretary, GRNMA, 2021, Available from: https://www.myjoyonline.com/789-nurses-and-midw ives-test-positive-for-covid-19-in-2021-general-secretary-grn $\mathrm{ma} /$ ?utm_source $=$ rss\&utm_medium $=$ rss\&utm_campaign $=789$ nurses-and-midwives-test-positive-for-covid-19-in-2021general-secretary-grnma.

[4] M. Kwofi, COVID-19 Hits 450 Doctors, kills 7, 2021, Available from: https://www.graphic.com.gh/news/general-news/covid -19-hits-450-doctors-kills-7.html.

[5] M. L. Manning, A. M. Gerolamo, M. A. Marino, M. E. Hanson-Zalot, and M. Pogorzelska-Maziarz, "COVID19 vaccination readiness among nurse faculty and student nurses," Nursing Outlook, 2021.

[6] WHO, COVID-19-Landscape of Novel Coronavirus Candidate Vaccine Development Worldwide, WHO, Geneva, Switzerland, 2021. 
[7] R. Dal-Ré, R. Stephens, and N. Sreeharan, "Let me choose my COVID-19 vaccine," European Journal of Internal Medicine, 2021.

[8] I. Jones, P. Roy, and V. Sputnik, "COVID-19 vaccine candidate appears safe and effective," The Lancet, vol. 397, no. 10275, pp. 642-643, 2021.

[9] R. Schraer, "COVID-19: Sputnik vaccine gives $92 \%$ protection in trail," 2021, Available from: https://www.bbc.com/news/ health-55900622\#:\%7E:text=Russia's\%20Sputnik\%20V\%20co ronavirus $\% 20$ vaccine,protection $\% 20$ against $\% 20$ hospitalisatio $\mathrm{n} \% 20$ and\%20death.

[10] J. Cohen, "Dosing debates, transparency issues roil vaccine rollouts," Science, vol. 371, no. 6525, pp. 109-110, 2021.

[11] S. P. Kaur and V. Gupta, "COVID-19 Vaccine: a comprehensive status report," Virus Research, vol. 288, Article ID 198114, 2020.

[12] R. Chou, T. Dana, D. I. Buckley, S. Selph, R. Fu, and A. M. Totten, "Epidemiology of and risk factors for coronavirus infection in health care workers," Annals of Internal Medicine, vol. 173, no. 2, pp. 120-136, 2020.

[13] N. N. Mahajan, A. Mathe, G. A. Patojar et al., "Prevalence, clinical presentations and treatment outcomes of COVID-19 among healthcare workers at a dedicated hospital in India," The Journal of the Association of Physicians of India, vol. 68, no. 12, pp. 16-21, 2020.

[14] J. Shaw, T. Stewart, K. B. Anderson et al., "Assessment of US health care personnel (HCP) attitudes towards COVID-19 vaccination in a large University health care system," Clinical Infectious Diseases, 2021.

[15] A. A. Dror, N. Eisenbach, S. Taiber et al., "Vaccine hesitancy: the next challenge in the fight against COVID-19," European Journal of Epidemiology, vol. 35, no. 8, pp. 775-779, 2020.

[16] P. Verger, D. Scronias, N. Dauby et al., "Attitudes of healthcare workers towards COVID-19 vaccination: a survey in France and French-speaking parts of Belgium and Canada, 2020," Eurosurveillance, vol. 26, no. 3, p. 2002047, 2021.

[17] M. K. Nzaji, L. K. Ngombe, G. N. Mwamba et al., "Acceptability of vaccination against COVID-19 among healthcare workers in the Democratic Republic of the Congo," Pragmatic and Observational Research, vol. 11, pp. 103-109, 2020.

[18] R. Shekhar, A. B. Sheikh, S. Upadhyay et al., "COVID-19 vaccine acceptance among health care workers in the United States," Vaccines, vol. 9, no. 2, p. 119, 2021.

[19] B. Roy, V. Kumar, and A. Venkatesh, "Health care workers' reluctance to take the Covid-19 vaccine: a consumer-marketing approach to identifying and overcoming hesitancy," NEJM Catalyst Innovations in Care Delivery, vol. 1, no. 6, 2020.

[20] K. Wang, E. L. Y. Wong, K. F. Ho et al., "Intention of nurses to accept coronavirus disease 2019 vaccination and change of intention to accept seasonal influenza vaccination during the coronavirus disease 2019 pandemic: a cross-sectional survey," Vaccine, vol. 38, no. 45, pp. 7049-7056, 2020.

[21] M. J. Deem, "Nurses' voices matter in decisions about dismissing vaccine-refusing families," AJN, American Journal of Nursing, vol. 118, no. 8, p. 11, 2018.

[22] A. K. Acheampong, M. Ganga-Limando, L. Aziato, and M. Ganga-Limando, "Perceived enablers of exclusive breastfeeding by teenage mothers in Ghana," South African Family Practice, vol. 62, no. 1, p. a5108, 2020.

[23] B. A. Aidam, R. Pérez-Escamilla, and A. Lartey, "Lactation counseling increases exclusive breast-feeding rates in Ghana," The Journal of Nutrition, vol. 135, no. 7, pp. 1691-1695, 2005.
[24] Ghana Health Service, The Health Sector in Ghana: Facts and Figures 2018, Ghana Health Service, Accra, Ghana, 2018.

[25] P. Geldsetzer, "Use of rapid online surveys to assess people's perceptions during infectious disease outbreaks: a crosssectional survey on COVID-19," Journal of Medical Internet Research, vol. 22, no. 4, Article ID e18790, 2020.

[26] S. Bostan, R. Erdem, Y. E. Öztürk, T. Kılıç, and A. Yılmaz, "The effect of COVID-19 pandemic on the Turkish society," Electronic Journal of General Medicine, vol. 17, no. 6, Article ID em237, 2020.

[27] L. Garrett, "COVID-19: the medium is the message," The Lancet, vol. 395, no. 10228, pp. 942-943, 2020.

[28] M. Barry, M.-H. Temsah, A. Alhuzaimi et al., "COVID-19 vaccine confidence and hesitancy among healthcare workers: a cross-sectional survey from a MERS-CoV experienced nation," 2020, https://www.medrxiv.org/content/10.1101/ 2020.12.09.20246447v1.full.

[29] K. O. Kwok, K. K. Li, W. I. Wei, K. H. Tang, S. Y. S. Wong, and S. S. Lee, "Are we ready when COVID-19 vaccine is available? Study on nurses' vaccine hesitancy in Hong Kong," 2020, https:// www.medrxiv.org/content/10.1101/2020.07.17.20156026v1.

[30] C. Fu, Z. Wei, S. Pei, S. Li, X. Sun, and P. Liu, "Acceptance and preference for COVID-19 vaccination in health-care workers (HCWs)," 2020, https://www.medrxiv.org/content/10.1101/ 2020.04.09.20060103v1.

[31] A. Gagneux-Brunon, M. Detoc, S. Bruel et al., "Intention to get vaccinations against COVID-19 in French healthcare workers during the first pandemic wave: a cross-sectional survey," Journal of Hospital Infection, vol. 108, pp. 168-173, 2021.

[32] A. Gadoth, M. Halbrook, R. Martin-Blais et al., "Assessment of COVID-19 vaccine acceptance among healthcare workers in Los Angeles," 2020, https://www.medrxiv.org/content/10. 1101/2020.11.18.20234468v1.full.

[33] L. Bunch, "A tale of two crises: addressing Covid-19 vaccine hesitancy as promoting racial justice," Hec Forum, pp. 1-12, 2021. 